\title{
Software and hardware improvement for the Streltsov ore field geodynamic testing area
}

\author{
Andrei Gladyr ${ }^{*}$, Vladimir Miroshnikov ${ }^{1}$ and Alexander Konstantinov ${ }^{1}$ \\ ${ }^{1}$ Mining Institute of Far Easten Branch of Russian Academy of Sciences, Khabarovsk, Russia
}

\begin{abstract}
For reliable forecasting and prevention of dangerous geodynamic phenomena in the Streltsov ore field area, the geodynamic testing area has been created. The geodynamic testing area includes modern measuring complexes that have different measurement methods and provide effective registration, and in-depth analysis of a wide range of geophysical wave fields changing in space and time. In the presence of anthropogenic seismicity, with the complication of the geomechanical and hydrological conditions of mining, the constant development of methods and means used at the geodynamic test site is required. It is necessary to install additional elements of a multi-level geomechanical monitoring system at sites in which dangerous geodynamic events are predicted and evidence of their reliable precursors. The modernization and integration of the measuring complexes of the geodynamic range into a multi-level geomechanical monitoring system allows to expand the controlled volume of the rock massif, to increase the quantity and quality of the information obtained about the state of the rock massif, which makes it possible to identify regional and local harbingers of dangerous dynamic phenomena and to justify a set of effective measures to reduce the level geodynamic risk improve the quality of forecast evaluation of geomechanical condition.
\end{abstract}

\section{Introduction}

At present, according to many experts, the influence of human activity on the earth surface state becomes more and more noticeable. Urbanization of territories, along with technogenic impact in the process of mining and processing of minerals, often leads to a significant deterioration of the overall environmental situation of the environment, climate change, activation of seismic processes [1-2].

In this connection, the role of creating specialized geodynamic testing areas on which complex (geodetic, geomechanical, engineering-seismological, engineering-seismometric, geophysical, etc.) observations of natural, technogenic and technogenic-induced geodynamic processes affecting the state of the techno-natural system is increasing. What is especially important for the areas that are subject to active man-caused impacts in the form of underground mining of minerals, construction of large engineering structures, reservoirs, etc. Emergency incidents in these areas can lead to catastrophic consequences for the

\footnotetext{
${ }^{*}$ Corresponding author: rush3112@mail.ru
} 
population as well as for the infrastructure of the facilities themselves and for the environment [3-5].

\section{Materials and methods}

In increasingly complex mining conditions, more in-depth complex geodynamic studies are needed, including: assessment of the geodynamic, seismic situation and stress-strain state of rock massifs, both within the Transbaikal block of the Amur plate, and in the area of the PAO "PPGHO" fields; studying regularities of geodynamic fields and processes in the field of technogenic influence of mining operations using seismic, seismoacoustic, geodetic, seismic and other methods to obtain fundamentally new information on the stresseddeformed state of the geological environment, as well as the identification of regional precursors of dynamic phenomena of different energy levels in mountainous arrays [6].

To solve the set tasks, a comprehensive program of $\mathrm{R} \& \mathrm{D}$ was developed in the period 2010-2016, the purpose of which was the creation of a multi-level complex geodynamic monitoring system ("Geodynamic testing area") in the Streltsov ore field.

At present the following systems are part of the geodynamic testing area [7]:

- geoacoustic monitoring system "Prognoz-ADS" (ASKGD) ("Antey" deposit, 34 geophones);

- a system of microseismic monitoring "Prognoz-S" (ASHSGD) (PUR No. 1, 12 seismic pavilions);

- system of deformation monitoring "SMD" (cages, places of possible deformations);

- laser scanning system "FARO FOCUS - 3D" (PUR No. 1, observation stations at 11, 12, 13 horizons);

- core drilling machine DIAMEK-232 (the core disk exploration method is the base one);

- portable impact control device "Prognoz-L" (local assessment of the state of the edge parts of the rock massif);

- laser deformometer (industrial site of PUR No. 1, IV horizon of mine No. 1);

- nanobarograf (industrial site of PUR No. 1, IV horizon of mine No. 1);

- regional network of GPS observations (the territory of Southern Transbaikalia);

- analytical program complex of mathematical modeling of the stress-strain state of rocks Sigma GT and ARGIS-10 (regional forecast of geodynamic activity);

- spatial model of deposits and mine workings based on the software product MINEFRAME (solving geotechnological problems and creating mining documentation).

At present, the team of Institute of Mining FEB RAS is actively working to modernize the geodynamic testing area facilities in order to improve its characteristics and improve the quality of the received information.

From 2016 to the present, the following works were planned and performed:

- expansion of the observation network for geomechanical processes;

- development of a special version of the elements of the system for working in explosion-proof design;

- modernization of software and hardware monitoring systems to increase the information and convenience of work;

- development of means for integrating the measuring means of the geodynamic test site into a single integrated observation network. 


\section{Results and discussion}

At the "Antey" deposit, related to the Streltsov ore field, 14 additional receiving transducers of the "Prognoz ADS" geoacoustic system were installed in the mine workings on the XIII and XIV horizons and the number of data transmission channels from 5 to 9 ports was expanded. On the XIII horizon, there is an underground apparatus room in the chamber opposite the mouth of the 6a-1302 ort and the gangway $6 a-1301$.

The installed sensors form several additional combinations of the receiving antenna and in combination with the existing geophones allow us to cover an even larger part of the minefield of the Antey deposit, including the riskiest areas of the massif near blocks 6a1006, 6a-1110-1102, 6a-1202 -1212, 6a-1312-1302 and 6a-1412, where dynamic manifestations of rock pressure have recently been observed.

For geomechanical monitoring under gas conditions, a set of geoacoustic equipment "Prognoz-ADS" in explosion proof design was developed. It included an explosion-proof switching and distribution unit consisting of cabinets and terminal boxes fixed to the frame. All elements on the explosion-proof frame with IP54 degree of protection are manufactured and certified by GORELTECH. In the main section (control cabinet) there are: an industrial computer with low power consumption (included in one network with the operator's computer, serves as an intermediate server for data collection of the system) complete with an external explosion-proof monitor and keyboard, a board with an RS-485 interface from MOXA; converter IMS-21GA-LX; the device of a filtration of sound channels and 4 devices of protection of information channels MOXA; power supply and time synchronization in the FPC model PSUt4.

Some elements of "Prognoz-ADS" developed in explosion-proof design and installed on an underground mine are shown in Fig. 1.

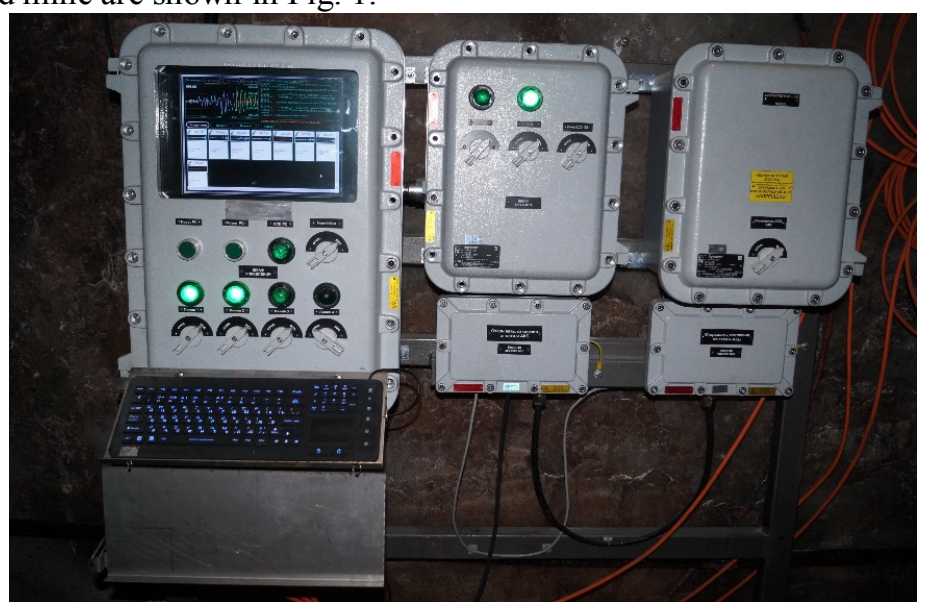

Fig. 1 - Explosion-proof switching and distribution unit "Prognoz-ADS" automated system (PB Ex d I Mb)

The network of microseismic observations in the area of the Streltsov ore field has been expanded by increasing the number of seismic pavilions of the microseismic system "Prognoz S" installed in underground workings on horizons V to XIV of the First Uranium Mining Management (PUR-1) from 8 to 12, which ensures greater reliability of the measuring complex and better accuracy of determining the parameters of seismic events in the controlled area of a rock mass with a volume of more than $12 \mathrm{~km}^{2}$.

To improve the efficiency and reliability of the microseismic system "Prognoz S", the technical and software tools have been modernized, including: the replacement of a central computing unit with a more modern one, built on the basis of the Intel NUC DE3815TYBE 
processor module; equipment of data acquisition modules by a specialized node - the watchdog timer WD021, which excludes "freezing" of equipment; development of special software for monitoring the state of the seismic station and the software shell of the operator's workplace with enhanced capabilities.

The software used in the system of microseismic monitoring can be divided into service, providing continuous collection of microseismic monitoring data and analytical, allowing to analyze and process the registered data (Fig. 2) [8-11].

To manage the process of interrogating the analog-to-digital converter, preliminary processing and subsequent transfer of information on the results of microseismic monitoring to the data collection server, software is developed and applied at each of the seismic stations. This software includes the following programs: the primary data server and the graphical interface of the data collection server.

\section{Primary data server}

The program is designed to collect data from primary converters of the microseismic monitoring system, preliminary processing, subsequent transmission of observation data and can be used as part of real-time geodynamic monitoring systems. To increase the speed and stability of the work, this program works in text mode.

The program provides the following functions:

- continuous data collection;

- the organization of a circular data buffer on an external medium with the ability to select data for an arbitrary time interval;

- digital filtering, decimation, compression of collected data;

- continuous transmission of processed data through a computer network for long-term storage and additional analysis.

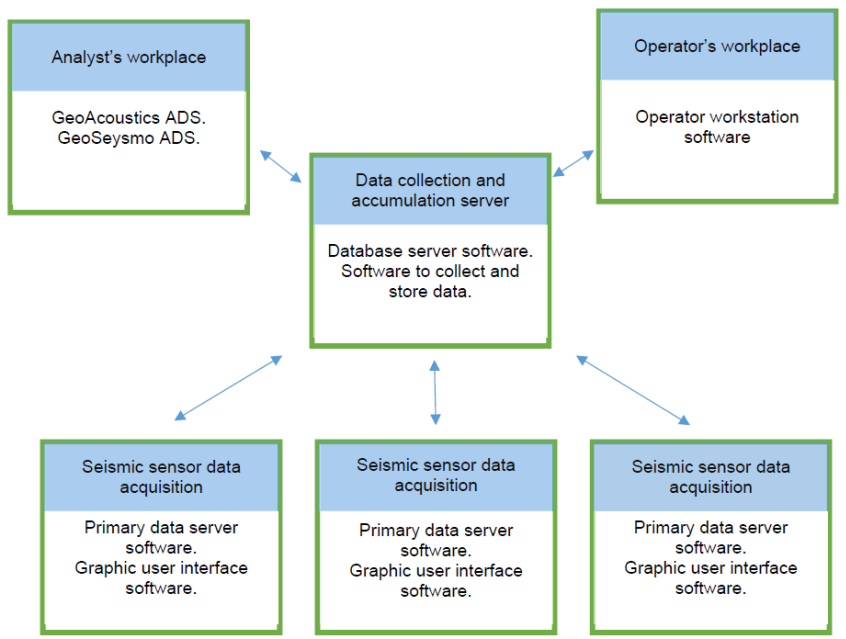

Fig. 2. Block diagram of the microseismic monitoring system software

\section{Graphical interface of the primary data server}

This program is designed to display information on the functioning of the primary data server of the microseismic monitoring system in real time and can be applied at each of the established seismic stations of the system to obtain up-to-date information on the process of collecting and transmitting the data of microseismic observations.

The program provides the following information:

- the operating status of the device, which displays information about the program version, the serial number of the analog-to-digital converter module, the number of active channels, the current sampling frequency, the operation modes of the program clock, the operation modes of the built-in digital filters. 
- graphical display of the digitized information coming from the analog-digital conversion module in real time.

- the protocol of the program, in which the protocols for sending the decimated data to the data collection server and isolating the potential microseismic pulses are displayed separately.

Simplified, the GUI of the primary data server is the "wrapper" for the primary data server. With this program, you can manage the primary data server and monitor its status. The program has three working screens: the first screen displays information about the device, mode of operation, status; The second screen contains graphs that show incoming signals from the sensor in real time; The third screen displays the protocol of the primary data server operation, including service and diagnostic information.

On the communication lines, the microseismic monitoring data is fed to the data collection and accumulation program.

\section{Data collection and accumulation program}

This program is designed to collect data from primary converters of the microseismic monitoring system, integrated into a single information network. The program performs preliminary processing of information on observations and is applied in the real-time monitoring system. To increase the speed and stability of the work, this program works in text mode.

The program provides the following functions:

- obtaining data (including decimated data) from the devices of primary converters, united in a single information network, through connection to data servers on each of the primary converters;

- detection in the received data of impulses on the set parameters;

- loading of the detected impulses of detailed data from all connected primary

converters and recording to an external medium for long-term storage.

\section{Workplace of the operator of the microseismic monitoring station}

This program interacts in real time with a program of sensor interrogation, preliminary processing of information and long-term storage of the data of the microseismic monitoring system, obtaining from it the necessary information for the generation of graphical information reports.

The program of the operator's automated workstation for the microseismic monitoring system allows:

- to expand the possibilities of visual display of the processes of information collection and processing;

- analyze the performance of each seismic post individually and the system as a whole for at least the last seven days;

- evaluate the performance of data channels in real time with memory for the last not less than seven days;

- to observe in real time the process of downloading registered microseismic pulses from seismic stations;

- view graphically the downloaded microseismic pulses registered previously.

Simplified, "Operator's seat of the microseismic monitoring station" is the "shell" for all service programs of the microseismic monitoring system. With this program, you can manage the operation of data collection modules and monitor the status of the process of collecting, transmitting and storing monitoring data for a long time.

The most important task for the generalization of experimental data characterizing the manifestations of seismoacoustic activity and technogenic seismicity in the conduct of underground works is the close integration of the applied systems into a single integrated observational network.

The integration process includes the following functionally completed steps [12]: 
- integration into the information exchange system (using interfaces and protocols USB, RS-485, RS-485, SHDSL with a transmission rate of up to $15.2 \mathrm{Mbps}$ );

- maintenance of common time management on all systems entering the geodynamic range (the implemented technical solutions ensure the accuracy of the system clock on each of the data collection servers is not less than $15 \mathrm{~ms}$ ) [13];

- realization of accumulation of monitoring data for geodynamic polygon systems in a single database;

- implementation of the ability to display and analyze monitoring data of geodynamic polygon systems using a single software package.

The main integrating platform of the geodynamic testing area is the software complex "Mineframe", intended for the complex solution of wide mining-geological and technological problems [14]. The three-dimensional graphic core, which is the basis of the software of the system, allows you to work with models of objects in a multi-window mode and provides a wide range of control options for displaying and editing objects of mining infrastructure.

\section{Conclusions}

To solve the problem of preventing dangerous manifestations of rock pressure in the underground mines of PAO "PPGHO" in the Streltsy ore field area, a multi-level system of complex geodynamic monitoring (complex geodynamic testing area) is created, which includes a number of complementary methods and technical means that ensure effective registration and in-depth analysis of the wide spectrum of spatially and time-varying parameters of geophysical wave fields (geoacoustic, microseismic, deformation ets).

The modernization allows to expand the controlled volume of the rock massif, improve the quality and quantity of information received and improve the quality of the predictive estimates of the geomechanical state.

Integration of geodynamic testing area systems into a single network of information exchange allows:

- manage the operation of any of the subsystems used from any workplace of the operator included in a single network, including those located in the surface complex;

- remote control and adjustment of acoustic emission, microseismic and deformation subsystems through a single Internet gateway;

- consolidate measurement data of geodynamic polygon subsystems on a single data server for centralized storage, processing and analysis, with the ability to automatically synchronize with remote data servers.

Integration of systems into a single network of providing the exact time allows us to trace with sufficient accuracy the correlation dependence between the indications of subsystems of microseismic, geoacoustic and deformation monitoring.

Joint interpretation of geoacoustic and microseismic monitoring data, correlated with geological and mining technical models, provides high reliability of forecast estimates.

The application of a multilevel system of geodynamic monitoring makes it possible to increase the quantity and quality of the information obtained about the state of the mountain massif, which makes it possible to identify regional and local harbingers of dangerous dynamic phenomena and to justify a set of effective measures to reduce the level of geodynamic risk in the development of mineral deposits in complex geological and shock hazard conditions.

The work was supported by the Russian Science Foundation (agreement No. 16-1700018). 


\section{References}

1. A.J. Mendecki, Seismic monitoring in mine. London: Chapmen and Hall (1997)

2. L. P. Ishukova, Uranium deposits of the Streltsov ore field in Transbaikalia (2007)

3. Aleksander J. Mendecki, Richard A. Lynch \& Dmitriy A. Malovichko. Routine MicroSeismic Monitoring in Mines. Australian Earthquake Engineering Society. 2010 Conference Perth, Western Australia, 33 (2010)

4. P.L. Swanson, Rockbursts and Seismicity in Mines - RaSiM5, South African Institute of Mining and Metallurgy, 11 (2001)

5. I. Yu. Rasskazov, B. G. Saksin, V. A. Petrov, B. A. Prosekin, Physical and technical problems of mineral development, 3, 3 (2012)

6. I. Yu. Rasskazov, A. V. Gladyr, P. A. Anikin, V. S. Svyatetskiy, B. A. Prosekin, Mining Journal, 8, 9 (2013)

7. A. V. Gladyr, D. S. Migunov, P. A. Anikin, G. A. Kalinov, V. I. Miroshnikov, Geomechanical and geotechnological problems of subsoil development in the North: Mining Information Analytical Bulletin, 11, 329 (2017)

8. I. Yu. Rasskazov, A. V. Gladyr, S. V. Deykin, D. O. Zhelnin, V. I. Miroshnikov, Certificate of registration of the computer program RUS 2014613073 (2014)

9. I. Yu. Rasskazov, A. V. Gladyr, S. V. Deykin, D. O. Zhelnin, E. A. Katella, Certificate of registration of the computer program RUS 2014613552 (2014)

10. A. V. Gladyr, Certificate of registration of the computer program RUS 2017660983 (2017)

11. A. V. Gladyr, Certificate of registration of the computer program RUS 2017660981 (2017)

12. A. V. Gladyr, Mining Information and Analytical Bulletin, 6, 220 (2017)

13. D. O. Zhelnin, A. V. Gladyr, E.A. Katella, S. V. Deykin, Problems of complex development of georesources Materials of the V All-Russian Scientific Conference with the participation of foreign scientists dedicated to the 30th anniversary of the Institute of Mining of the Far Eastern Branch of the Russian Academy of Sciences and The 100th anniversary of his birth. corr. RAS E. I. Bogdanova, 49 (2013)

14. S. V. Lukichev, O. V. Nagovitsyn, I. E. Semenova, O..V. Belogorodtsev, Mining Journal, 8, 53 (2015) 